is to decide the best indication. We are in the midst of animal studies for IL-34 antagonists," Williams told SciBX.

\section{The target}

\section{FivePrime's time}

\section{By Lauren Martz, Staff Writer}

A paper published in Science has identified the previously unknown protein IL-34 as being involved in monocyte proliferation. ${ }^{1}$ Besides opening new doors for autoimmune disease and cancer research, the finding provides proof of concept for the secreted protein discovery platform of Five Prime Therapeutics Inc., a company that has been running quietly since raising $\$ 45$ million to fund preclinical research in 2005.

FivePrime was founded with the goal of industrializing secreted protein discovery and development. Its technological platform includes a library of secreted proteins and extracellular domains of transmembrane proteins, and assays that measure the metabolic, growth and transcriptional responses of each protein. Analysis of the assay data allows the company to select proteins with specificity for desired assays and thus for desired properties.

In the Science paper, Lewis Williams and colleagues put this discovery engine to work to identify a protein that regulates monocytes with the hope of finding a protein that could be targeted to treat cancer or autoimmune disease. Monocytes mature to form macrophages and are essential for the immune system's recognition and destruction of foreign bodies. Monocytes are thus a line of defense against tumor cells, whereas improper monocyte activation can cause autoimmune diseases. ${ }^{2}$

Williams, the corresponding author on the paper, is cofounder and chairman of FivePrime. He is also an adjunct professor at the Cardiovascular Research Institute of the University of California, San Francisco.

The team scanned about 3,400 secreted proteins and extracellular domains in 25 assays and selected IL-34 for further research after functional studies showed that purified IL-34 promoted monocyte viability. An assay measuring DNA replication showed the enhanced viability was due to cell proliferation rather than just increased survival. In human bone marrow cultures, IL-34 promoted formation of a macrophage precursor, which further supports the cytokine's role in monocyte maturation.

Researchers also found that this previously unknown growth factor is expressed in a variety of cell types including cardiac, brain, lung, liver, kidney, spleen, thymus, testes, ovary, small intestine, prostate and colon.

"Because we are dealing with a ligand expressed in such a range of cell types that acts on a receptor with very broad potential clinical applications, the most critical next step in pursuing IL-34 as a therapeutic target
Although IL-34 is a newly discovered protein, additional results reported in the Science paper reveal that its receptor is not. Through a subsequent screening of 858 extracellular protein domains, the researchers identified IL-34's receptor as the previously described and well-studied colonystimulating factor-1 receptor (CSF-1R).

Until now, CSF-1, the primary regulator of the development, survival, proliferation and differentiation of macrophages, was the only known ligand for CSF-1R. ${ }^{3}$ The new findings raise the question of whether targeting IL-34 would have benefits over targeting CSF-1.

Richard Stanley, chairman of the Department of Developmental and Molecular Biology at the Albert Einstein College of Medicine, told SciBX the CSF-1 ligand/receptor pair has been implicated in the regulation of innate immunity, and activation of the receptor might be associated with development of atherosclerosis, osteoporosis, kidney allograft rejection, collageninduced arthritis, leukemia and obesity. Thus, he said, antagonists of the receptor could have broad therapeutic potential.

Steven Bass, senior director of R\&D at Maxygen Inc., told SciBX that a new ligand for CSF$1 \mathrm{R}$ is a welcome addition to the pool of potential therapeutics. "CSF-1R is a proto-oncogene. As such, you might want to block IL-34" to treat cancer, he said.

Maxygen's MAXY-G34 is a next-generation G-CSF that is in Phase II testing to treat chemotherapy-induced neutropenia in breast cancer patients.

Heishan Lin, first author on the paper and director of immunology at FivePrime, agreed that cancer is indeed an area where IL-34's regulation of macrophage colony-forming cell development could have therapeutic potential. Lin also noted that IL-34 "can give rise to osteoclasts, which are relevant to osteoporosis, and microglia, which are implicated in neurological disorders including multiple sclerosis."

Stanley cautioned that although both ligands, IL-34 and CSF-1, act through the same receptor, much more needs to be known about IL-34 before it is considered a valid therapeutic target. "We know IL-34 acts on the CSF-1 receptor. Now what we really need to know is: does it only act on that receptor, and are there still other ligands acting on that receptor as well," he said.

Previous studies in mice showed that knockout of CSF-1 did not cause phenotypic effects as severe as those caused by knockout of its receptor. ${ }^{4}$ With IL-34 in hand, Stanley suggested that "the critical next step is to test whether a double knockout of CSF-1 and IL-34 has the same phenotypic effects as the CSF-1R knockout model. If this is the case, you confirm that these are the two primary ligands acting on the receptor and help rule out the possibility that IL-34 is acting on other receptors, which could cause side effects." 


\section{TARGETS \& MECHANISMS}

He added, "We understand how CSF-1 and IL-34 are the same; now what we need to understand is how the two differ. We want to find out where their expression patterns do not overlap and how they are differentially regulated."

This is important because "if there is a big overlap, there may be little advantage to targeting IL-34, but if it has selective effects, which are very likely, it could be useful," said Stanley. For example, if inhibition of CSF$1 \mathrm{R}$ has side effects, it could be therapeutically advantageous to target its ligands individually.

\section{Where to next?}

Whatever the final fate of IL-34 as a drug target, the Science paper has demonstrated that FivePrime's comprehensive discovery platform will most likely be useful for identifying additional therapeutic targets. "Being able to find a novel cytokine that has been hiding under our noses is impressive," said Bassil Dahiyat, CEO of Xencor Inc.

Dahiyat added that an advantage of the platform is its use of cell-based assays rather than binding or panning assays, "which give much less information and are so much more difficult to deduce relevant biology from." Dahiyat and Stanley both told SciBX that increasing the number of functional assays would further increase the success of protein discovery platforms such as FivePrime's.

Xencor's Protein Design Automation (PDA) technology is used to optimize antibodies for cancer and therapeutic proteins for inflammation and autoimmune disorders. The company's XmAb 2513, a humanized monoclonal antibody against CD30, is in Phase I testing to treat cancer.

In addition to the IL-34 paper in Science, FivePrime's discovery engine received a further validation boost because of last week's deal with Pfizer Inc. to discover antibody targets and protein therapeutics to treat cancer and diabetes. The biotech said the deal does not include IL-34.

Since 2005, Williams said, "we've moved a compound near IND filing, have three additional preclinical development candidates, a number of other potential lead molecules, a new in vivo screening platform and have expanded the company to about 85 individuals."

He told SciBX that lead compound FP-1039, an extracellular domain molecule to treat cancer, is within a few weeks of an IND submission.

\section{REFERENCES}

1. Lin, H. et al. Science; published online May 9, 2008; doi:10.1126/science. 1154370

Contact: Lewis T. Williams, Five Prime Therapeutics Inc., San Francisco, Calif.

e-mail: rusty.williams@fiveprime.com

2. Mosser, D. J. Leukoc. Biol. 73, 209-212 (2003)

3. Wei, S. et al. J. Leukoc. Biol. 80, 14-45 (2006)

4. Dai, X. M. et al. Blood 99, 111-120 (2002)

COMPANIES AND INSTITUTIONS MENTIONED

Albert Einstein College of Medicine, Bronx, N.Y. Five Prime Therapeutics Inc., San Francisco, Calif. Maxygen Inc. (NASDAQ:MAXY), Redwood City, Calif. Pfizer Inc. (NYSE:PFE), New York, N.Y.

University of California, San Francisco, Calif.

Xencor Inc., Monrovia, Calif. 